International Electronic Journal of Algebra

Volume 22 (2017) 28-38

DOI: $10.24330 /$ ieja. 325918

\title{
A GENERALIZATION OF TOTAL GRAPHS OF MODULES
}

\author{
Ahmad Abbasi and Leila Hamidian Jahromi \\ Received: 15 September 2016; Revised: 15 June 2017 \\ Communicated by Sarah Glaz
}

\begin{abstract}
Let $R$ be a commutative ring, and let $M \neq 0$ be an $R$-module with a non-zero proper submodule $N$, where $N^{\star}=N-\{0\}$. Let $\Gamma_{N^{\star}}(M)$ denote the (undirected) simple graph with vertices $\left\{x \in M-N \mid x+x^{\prime} \in N^{\star}\right.$ for some $\left.x \neq x^{\prime} \in M-N\right\}$, where distinct vertices $x$ and $y$ are adjacent if and only if $x+y \in N^{\star}$. We determine some graph theoretic properties of $\Gamma_{N^{\star}}(M)$ and investigate the independence number and chromatic number.
\end{abstract}

Mathematics Subject Classification (2010): 13C99, 05C69

Keywords: Commutative ring, total graph, independence number

\section{Introduction}

Throughout, all rings are commutative with non-zero identity and all modules are unitary. Let $R$ be a ring, $M \neq 0$ an $R$-module, and $N$ a non-zero proper submodule of $M$. The total graph of a commutative ring $R$, denoted by $T(\Gamma(R))$, was introduced by Anderson and Badawi in [3], as the graph with all elements of $R$ as vertices, and two distinct vertices $x, y \in R$ are adjacent if and only if $x+y \in Z(R)$, where $Z(R)$ denotes the set of zero-divisors of $R$. The concept of total graphs is a great concept that is usually used in commutative algebra to obtain many interesting graphs in this field. In [1] and [2], A. Abbasi and S. Habibi, gave a generalization of the total graph. They studied in [2] the total graph $T\left(\Gamma_{N}(M)\right)$ of a module $M$ over a commutative ring with respect to a proper submodule $N$. It is an undirected graph with the vertex set $M$, where two distinct vertices $m$ and $n$ are adjacent if and only if $m+n \in M(N)$, where $M(N)=\{m \in M \mid r m \in N$ for some $r \in R-(N: M)\}$. It is easy to see that $M(N)$ is closed under multiplication by scalars. However, $M(N)$ may not be an additive subgroup of $M$. Here we introduce a generalization of total graphs, denoted by $\Gamma_{N^{\star}}(M)$, as the (undirected) simple graph with vertices $\left\{x \in M-N \mid x+x^{\prime} \in N^{\star}\right.$ for some $\left.x \neq x^{\prime} \in M-N\right\}$, where distinct vertices $x$ and $y$ are adjacent if and only if $x+y \in N^{\star}=N-\{0\}$.

Let $G$ be a simple graph. If there is a path from any vertex to any other vertex of graph $G$, then $G$ is said to be connected, and $G$ is said to be totally disconnected 
if there is no path connecting any pair of vertices. For vertices $x_{1}$ and $x_{2}$ of $G$, we define $d\left(x_{1}, x_{2}\right)$ to be the length of a shortest path between $x_{1}$ and $x_{2}$ $\left(d(x, x)=0\right.$ and $d\left(x_{1}, x_{2}\right)=\infty$ if there is no such path). The diameter of $G$ is $\operatorname{diam}(G)=\sup \left\{d\left(x_{1}, x_{2}\right) \mid x_{1}\right.$ and $x_{2}$ are vertices of $\left.G\right\}$. The girth of $G$, denoted by $\operatorname{gr}(G)$, is the length of its shortest cycle; $\operatorname{gr}(G)=\infty$ if $G$ contains no cycles, in this case, $G$ is called an acyclic graph. A complete graph is one which every two vertices are adjacent. A complete graph with $n$ vertices is denoted by $K^{n}$. A bipartite graph $G$ is a graph whose vertex set $V(G)$ can be partitioned into disjoint subsets $U_{1}$ and $U_{2}$ in such a way that each edge of $G$ has one end vertex in $U_{1}$ and the other in $U_{2}$. In particular, if $E$ consists of all possible such edges, then $G$ is called a complete bipartite graph and is denoted by $K^{m, n}$ when $\left|U_{1}\right|=m$ and $\left|U_{2}\right|=n$. For a vertex $v$ of $G, \operatorname{deg}(v)$ denotes the degree of $v$ and we set $\delta(G):=\min \{\operatorname{deg}(v)$ : $v$ is a vertex of $G\}$. A graph $G$ is called k-regular if every vertex has degree $k$. A subgraph of $G$ is the graph formed by a subset of the vertices and edges of $G$. Two subgraphs $G_{1}$ and $G_{2}$ of $G$ are said to be disjoint if $G_{1}$ and $G_{2}$ have no common vertices and no vertex of $G_{1}$ (resp., $G_{2}$ ) is adjacent (in $G$ ) to any vertex not in $G_{1}$ (resp., $\left.G_{2}\right)$. The union of two graphs $G_{1}=\left(V_{1}, E_{1}\right)$ and $G_{2}=\left(V_{2}, E_{2}\right)$ is the graph $G_{1} \cup G_{2}$ whose vertex set is $V_{1} \cup V_{2}$ and whose edge set is $E_{1} \cup E_{2}$. A complete subgraph of $G$ is called a clique. The clique number, $\omega(G)$, is the greatest integer $n \geq 1$ such that $K^{n}$ is a subgraph of $G$, and $\omega(G)=\infty$ if $K^{n} \subseteq G$ for all $n \geq 1$. A matching in a graph $G$ is a set of edges such that no two have a vertex in common. A spanning matching of a graph is said to be a perfect matching. A star graph $S_{k}$ is the complete bipartite graph $K^{1, k}$. A Hamiltonian cycle is a cycle that visits each vertex exactly once. A graph that contains a Hamiltonian cycle is called a Hamiltonian graph. A walk is an alternating sequence of vertices and edges which are incident, that begins and ends with a vertex. A tour is a closed walk that traverses each edge at least once. An Eulerian tour in an undirected graph is a tour that traverses each edge exactly once. If such a tour exists, the graph is called Eulerian. A connected component (or just component) of an undirected graph is a maximal connected induced subgraph. An independent set is a set of vertices in a graph, no two of which are adjacent. That is, it is a set $\mathrm{S}$ of vertices such that for every two vertices in $\mathrm{S}$, there is no edge connecting the two. The vertex independence number of $G$, often called the independence number, is the cardinality of a largest independent vertex set, i.e., the maximum size among all independent vertex sets of $G$. The independence number is denoted by $\alpha(G)$. A vertex cover of $G$ is a set of vertices such that each edge of $G$ is incident to at least one vertex of the set. The vertex cover number is the minimum size 
among all vertex covers in the graph, denoted by $\beta(G)$. A coloring of a graph is a proper (vertex) coloring with colors such that no two vertices sharing the same edge have the same color. A coloring using $k$ colors is called a (proper) $k$ coloring. The smallest number of colors needed to color the vertices of $G$ is called its chromatic number and is denoted by $\chi(G)$.

The main objective of this paper is to study some properties of the graph $\Gamma_{N^{\star}}(M)$. We also investigate the independence number and chromatic number of the graph $\Gamma_{N^{\star}}(M)$.

\section{Properties of $\Gamma_{N^{\star}}(M)$}

In this section, we investigate some properties of $\Gamma_{N^{\star}}(M)$. Throughout, $N$ is a non-zero proper submodule of a non-zero $R$-module $M$, where $R$ is commutative ring.

Definition 2.1. Let $R$ be a commutative ring, $M$ be an $R$-module, $N$ be a submodule of $M$, and let $N^{\star}=N-\{0\}$. We define an undirected simple graph $\Gamma_{N^{\star}}(M)$ with vertices $\left\{x \in M-N \mid x+x^{\prime} \in N^{\star}\right.$ for some $\left.x \neq x^{\prime} \in M-N\right\}$, where distinct vertices $x$ and $y$ are adjacent if and only if $x+y \in N^{\star}$.

Remark 2.2. (1) $x \in V\left(\Gamma_{N^{*}}(M)\right)$ if and only if $N(x) \neq \emptyset$, where $N(x)=$ $\left\{x^{\prime} \in V\left(\Gamma_{N^{\star}}(M)\right) \mid x^{\prime} \neq x, x+x^{\prime} \in N^{\star}\right\}$. So, there is no isolated vertex in $\Gamma_{N^{*}}(M)$. In particular, $\Gamma_{N^{*}}(M)$ is not totally disconnected.

(2) Let $x, y \in V\left(\Gamma_{N^{\star}}(M)\right)$ be adjacent with $x-y \in N^{\star}$, then $x+y-x+y \in N$ and $x+y+x-y \in N$; so $2 x, 2 y \in N$.

(3) Let $x, y \in V\left(\Gamma_{N^{\star}}(M)\right)$ with $x \neq y$ and $N(x) \cap N(y) \neq \emptyset$. Then $x-y \in N^{\star}$.

(4) $\Gamma_{N^{*}}(M)$ is a perfect matching if and only if for all $x, y \in V\left(\Gamma_{N^{*}}(M)\right)$ with $x \neq y$, one has $N(x) \cap N(y)=\emptyset$ or $|N(x)|=|N(y)|=1$.

Example 2.3. Let $M=\mathbb{Z}_{12}$ and $N=\{0,4,8\}$. Then $\Gamma_{N^{\star}}(M)$ has the following form:

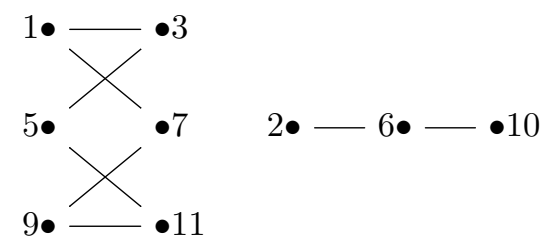

Figure 1.

Theorem 2.4. If $x, y \in V\left(\Gamma_{N^{\star}}(M)\right)$ are distinct vertices connected by a path of length 3 with $x+y \neq 0$, then $x, y$ are adjacent. 
Proof. Let $x, m_{1}, m_{2}, y$ be distinct vertices of $\Gamma_{N^{\star}}(M)$ with a path $x-m_{1}-m_{2}-y$. Since $x+m_{1}, m_{1}+m_{2}$ and $m_{2}+y \in N^{\star}$, we have $x+y=\left(x+m_{1}\right)+\left(y+m_{2}\right)-\left(m_{1}+\right.$ $\left.m_{2}\right) \in N$. This yields $x+y \in N^{*}$, since $x+y \neq 0$; so $x$ and $y$ are adjacent.

Corollary 2.5. Let $e=x x^{\prime}$ and $f=y y^{\prime}$ be edges of $\Gamma_{N^{*}}(M)$, where the sum of each end point of $e$ and each end point of $f$ does not equal zero. If two end points of $e$ and $f$ are adjacent, then so are the other two.

Proof. Without loss of generality, we may assume that $x^{\prime}$ and $y$ are adjacent; so there is a path $x-x^{\prime}-y-y^{\prime}$ in $\Gamma_{N^{*}}(M)$. Therefore, $x$ and $y^{\prime}$ are adjacent, by Theorem 2.4.

Remark 2.6. In Corollary 2.5, the condition "does not equal zero" is necessary. For instance, in Example 2.3, set $x=5, y=9, x^{\prime}=3, y^{\prime}=11$. Then $x$ and $y^{\prime}$ are adjacent, but $x^{\prime}$ and $y$ are not.

Theorem 2.7. If $x, y \in V\left(\Gamma_{N^{*}}(M)\right)$ are distinct vertices connected by a path of length 4 , then there exists a path of length 2 between them. In particular, there is $t \in V\left(\Gamma_{N^{\star}}(M)\right)$ with $t \in N(x) \cap N(y)$.

Proof. Let $x, m_{1}, m_{2}, m_{3}, y$ be distinct vertices of $\Gamma_{N^{\star}}(M)$ with a path $x-m_{1}-$ $m_{2}-m_{3}-y$. If $x+m_{3} \neq 0$ or $m_{1}+y \neq 0$, then $x$ and $m_{3}$, or $y$ and $m_{1}$, are adjacent by Theorem 2.4, as we desired. So let $x=-m_{3}$ and $y=-m_{1}$. Then we have a path $x\left(=-m_{3}\right)-m_{1}-m_{2}-m_{3}-y\left(=-m_{1}\right)$. Thus, $x\left(=-m_{3}\right)-\left(-m_{2}\right)-y(=$ $\left.-m_{1}\right)$ is a path of length 2 .

Theorem 2.8. Let $\Gamma_{N^{\star}}(M)$ be connected. If $t_{1}-t_{2} \in N^{\star}$ for all adjacent vertices $t_{1}$ and $t_{2}$ of $\Gamma_{N^{\star}}(M)$, then $\operatorname{diam}\left(\Gamma_{N^{\star}}(M)\right) \in\{1,2\}$.

Proof. For every path of length 3 such as $x-m_{1}-m_{2}-y$ in $\Gamma_{N^{*}}(M)$, if $x+y \neq 0$, then $x$ and $y$ are adjacent by Theorem 2.4 and $\operatorname{diam}\left(\Gamma_{N^{\star}}(M)\right) \leq 2$. Let $x=-y$. Then there is a path $x(=-y)-m_{1}-m_{2}-y$. Our hypothesis yields $x-m_{2}$, and we are done.

Theorem 2.9. $\operatorname{diam}\left(\Gamma_{N^{*}}(M)\right) \in\{1,2,3, \infty\}$. In particular, if $\Gamma_{N^{\star}}(M)$ is connected, then $\operatorname{diam}\left(\Gamma_{N^{\star}}(M)\right) \leq 3$.

Proof. By Theorem 2.7, we can reduce every path of length greater than 3 to a path of length at most 3 . 
Example 2.10. Let $M=\mathbb{Z}_{8}$ and $N=\{0,2,4,6\}$. Then $\Gamma_{N^{*}}(M)$ has the following form:

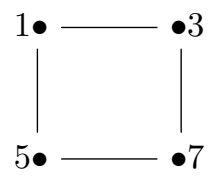

Figure 2.

Theorem 2.11. Let $\Gamma_{N^{\star}}(M)$ be connected. Then it is complete if and only if $2 t=0$ for every $t \in V\left(\Gamma_{N^{*}}(M)\right)$.

Proof. Suppose that $\Gamma_{N^{\star}}(M)$ is complete and $2 t \neq 0$ for some $t \in V\left(\Gamma_{N^{\star}}(M)\right)$. Then $-t$ is a vertex and $0=t+(-t) \in N^{\star}$, which is a contradiction. Suppose $2 t=0$ for all vertices $t$. Then $\operatorname{diam}\left(\Gamma_{N^{\star}}(M)\right) \leq 3$ by Theorem 2.9. Let $x-t-y$ be a path in $\Gamma_{N^{\star}}(M)$. Then by part (3) of Remark 2.2, $x+y \in N^{\star}$ (since by assumption $2 y=0$ implies that $y=-y)$. Let $d(x, y)=3$. So there is a path $x-t_{1}-t_{2}-y$ in $\Gamma_{N^{*}}(M)$. If $x+y=0$, then $x=-y=y$ (since $2 y=0$ ); this contradicts our assumption, so $x+y \neq 0$. Hence, $x$ and $y$ are adjacent by Theorem 2.4. So, $\Gamma_{N^{*}}(M)$ is complete.

Theorem 2.12. If $2 x \neq 0$ for every $x \in V\left(\Gamma_{N^{\star}}(M)\right)$, then $\operatorname{gr}\left(\Gamma_{N^{\star}}(M)\right) \in\{3,4,6, \infty\}$.

Proof. (1) It is clear that $\Gamma_{N^{\star}}(M)$ has more than two vertices. For all $x \in$ $V\left(\Gamma_{N^{\star}}(M)\right)$, let $|N(x)|=1$. Then $\operatorname{gr}\left(\Gamma_{N^{\star}}(M)\right)=\infty$, since in this case $\Gamma_{N^{\star}}(M)$ is just a perfect matching.

(2) Suppose there is $t \in V\left(\Gamma_{N^{\star}}(M)\right)$ such that $|N(t)| \geq 2$.

$\left(1^{\prime}\right)$ If for all vertices $t$ with $|N(t)| \geq 2$, we have $|N(x)|=1$ for every $x \in N(t)$, then there are not any cycles in $\Gamma_{N^{\star}}(M)$.

$\left(2^{\prime}\right)$ Suppose there exists $y \in N(t)$ such that $|N(y)| \geq 2$ and this condition is satisfied just for $y$. There is an $x \in N(t)$ such that $|N(x)|=1$, since $|N(t)| \geq$ 2. If $x \neq-y$, then by part 3 of Remark 2.2 , one has $(-y)-x-t-y$ and if $x=-y$, then $(-y)-t-y-m$ for some $m \in N(y)$. This implies that $(-m)-(-y)-t-y-m$, which contradicts $|N(x)|=1$. So, we should have at least two vertices $x, y \in N(t)$ such that $|N(x)|,|N(y)| \geq 2$. If $x$ and $y$ are adjacent, then $x-t-y-x$ and $\operatorname{gr}\left(\Gamma_{N^{*}}(M)\right)=3$. We assume that $x$ and $y$ are not adjacent for every $x, y \in N(t)$.

(a) Let $|N(x) \cap N(y)|=1$. If $2 t \in N^{\star}$, then $x+t, y+t \in N^{\star}$; so $x+y+2 t \in N$. This yields $x+y \in N$ and so $x+y=0$ (since $x$ and $y$ are not adjacent). Therefore, $x=-y$ and there exists a path $(-y)-t-y-(-t)$. This implies that $-y$ and 
$-t$ are adjacent. So, $-t \in N(x) \cap N(y)$ where contradicts our assumption, since $|N(x) \cap N(y)|=1$.

Now, assume that $2 t \notin N^{\star}$ and $|N(t)|=2$. There is a path $(-y)-x-t-y$ $-(-x)$ in $\Gamma_{N^{\star}}(M)$ by part 3 of Remark 2.2 ; so $(-t)-(-y)-x-t-y-(-x)$ and $-t$ and $-x$ are adjacent. Hence $\operatorname{gr}\left(\Gamma_{N^{\star}}(M)\right) \leq 6$. If $|N(t)| \geq 3$, there is a path $m-t-y-(-x)$ for some vertex $m \neq x$. Since $m-x \neq 0$, one has $\operatorname{gr}\left(\Gamma_{N^{\star}}(M)\right) \leq 4$, by Theorem 2.4. If $-x, t$ are adjacent then there is a path $(-x)-t-y-(-x)$ and $\operatorname{gr}\left(\Gamma_{N^{\star}}(M)\right)=3$.

(b) Let $|N(x) \cap N(y)| \geq 2$. There is a path $m-x-t-y-m$. Hence $\Gamma_{N^{\star}}(M)$ contains a 4 -cycle and $\operatorname{gr}\left(\Gamma_{N^{*}}(M)\right) \leq 4$.

Corollary 2.13. $\Gamma_{N^{*}}(M)$ is an acyclic graph if and only if it is a disjoint union of some star components.

Proof. Suppose that graph $\Gamma_{N^{\star}}(M)$ is an acyclic graph. If $\Gamma_{N^{\star}}(M)$ has a non-star component, then there exists at least one path of length 3 as $x-t_{1}-t_{2}-y$ in $\Gamma_{N^{\star}}(M)$. We assumed that $\Gamma_{N^{\star}}(M)$ is an acyclic graph, so $x+y=0$, by Theorem 2.4. Hence, we have a path $\left(-t_{2}\right)-x-t_{1}-t_{2}-y$. Theorem 2.7 yields there is a cycle in $\Gamma_{N^{*}}(M)$, which contradicts our assumption. Hence all paths are of lengths 1 or 2 . This implies that all components are in the form of stars.

Theorem 2.14. The following statements hold for the clique number of $\Gamma_{N^{\star}}(M)$.

(1) $\omega\left(\Gamma_{N^{*}}(M)=2\right.$ if $N(x) \cap N(y)=\emptyset$ for every distinct $x, y \in V\left(\Gamma_{N^{*}}(M)\right)$.

(2) If there exist adjacent vertices $x$ and $y$ in $\Gamma_{N^{*}}(M)$ such that $N(x) \cap N(y) \neq$ $\emptyset$, then $\omega\left(\Gamma_{N^{\star}}(M)\right) \geq 3$.

(3) If $2 t=0$ for all $t \in V\left(\Gamma_{N^{*}}(M)\right)$ and there are adjacent vertices $x$ and $y$ in $\Gamma_{N^{*}}(M)$ such that $x^{\prime}+y^{\prime} \neq 0$ for some $x^{\prime} \in N(x)$ and $y^{\prime} \in N(y)$, then $\omega\left(\Gamma_{N^{\star}}(M)\right) \geq 4$.

Proof. (1) It is clear, since $\Gamma_{N^{\star}}(M)$ is a perfect matching.

(2) It is clear, since there is a triangular cycle.

(3) There is a path $x^{\prime}-x-y-y^{\prime}$ in $\Gamma_{N^{\star}}(M)$. In view of Theorem 2.4, $x^{\prime}$ and $y^{\prime}$ are adjacent. So, $x^{\prime}, y$ and $x, y^{\prime}$ are adjacent by part 3 of Remark 2.2. Hence $\omega\left(\Gamma_{N^{\star}}(M) \geq 4\right.$.

Definition 2.15. (See [5, Definition 2.9]) Let $m \in M-N$. We call the subset $m+N^{\star}$ a column of $\Gamma_{N^{\star}}(M)$. If $2 m \in N^{\star}$ for every $m \in M-N$, then we call $m+N^{\star}$ a connected column of $\Gamma_{N^{\star}}(M)$.

Theorem 2.16. Suppose $\Gamma_{N^{\star}}(M)$ contains at least one connected column and $\left|N^{\star}\right| \geq 4$ with $2 m \neq 0$ for every $m \in N^{\star}$. Then $\operatorname{gr}\left(\Gamma_{N^{\star}}(M)=3\right.$. 
Proof. Let $x+N^{\star}$ be a connected column in $\Gamma_{N^{\star}}(M)$. Then $2 x \in N^{\star}$. Let $n \neq 2 x,-2 x$ in such a way that $n \in N^{\star}$. Then $x-(x+n)-(x-n)-x$ is a cycle of length 3 in $\Gamma_{N^{\star}}(M)$.

Recall that a vertex $x$ of a connected graph $G$ is called a cut-point of $G$ if there are vertices $u, w$ of $G$ such that $x$ lies on every path from $u$ to $w$ (with $x \neq u$, $x \neq w)$. Equivalently, for a connected graph $G, x$ is called a cut-point of $G$ if $G-\{x\}$ is not connected.

Theorem 2.17. Let $\Gamma_{N^{\star}}(M)$ be connected with $2 x \neq 0$ for all $x \in V\left(\Gamma_{N^{*}}(M)\right)$. Then $\Gamma_{N^{\star}}(M)$ has no cut-points.

Proof. Assume the vertex $x$ of $\Gamma_{N^{\star}}(M)$ is a cut-point. Then there exist vertices $u, w$ of $\Gamma_{N^{\star}}(M)$ such that $x$ lies on every path from $u$ to $w$ (therefore, $x \neq u, w$ ). By Theorem 2.9, the shortest path from $u$ to $w$ is of length 2 or 3 .

Case 1. Suppose $u-x-w$ is a path of shortest length from $u$ to $w$. There is a path $(-w)-u-x-w-(-u)$ in $\Gamma_{N^{\star}}(M)$. So there exists a path $u-(-w)-(-x)$ - $(-u)-w$ by part 3 of Remark 2.2, which contradicts our assumption.

Case 2. Suppose (without loss of generality) that $u-x-y-w$ is a path of shortest length from $u$ to $w$ in $\Gamma_{N^{*}}(M)$. Therefore, $N(u) \cap N(w)=\emptyset$. Since $u$ and $w$ are not adjacent, by Theorem 2.4, we have $u+w=0$ and $(-y)-u(=$ $-w)-x-y-w$. So, there exists a path $u-(-y)-(-x)-w$, which contradicts our assumption.

Remark 2.18. Suppose there is a path as $u-t-w$ in $\Gamma_{N^{\star}}(M)$ such that $|N(u)|=$ $|N(w)|=1$. Then $\Gamma_{N^{\star}}(M)$ has a cut-point.

Theorem 2.19. The degree of every vertex $x$ of $\Gamma_{N^{\star}}(M)$ is either $\left|N^{\star}\right|$ or $\left|N^{\star}\right|-1$. In particular, if $2 m \in N^{\star}$ for every vertex $m$ of $\Gamma_{N^{\star}}(M)$, then $\Gamma_{N^{\star}}(M)$ is a $\left|N^{\star}\right|-1$ regular graph.

Proof. Let $x \in V\left(\Gamma_{N^{\star}}(M)\right)$. If $x$ is adjacent to $y$, then $x+y=a \in N^{\star}$ and hence, $y=a-x$ for some $a \in N^{\star}$. There are two cases:

Case 1. Suppose that $2 x \in N^{\star}$. Then $x$ is adjacent to $a-x$ for every $a \in$ $N^{\star}-\{2 x\}$. Thus the degree of $x$ is $\left|N^{\star}\right|-1$. In particular, if $2 m \in N^{\star}$ for every $m \in V\left(\Gamma_{N^{\star}}(M)\right)$, then $\Gamma_{N^{\star}}(M)$ is a $\left|N^{\star}\right|$ - 1-regular graph.

Case 2. Suppose that $2 x \notin N^{\star}$. Then $x$ is adjacent to $a-x$ for all $a \in N^{\star}$. Thus the degree of $x$ is $\left|N^{\star}\right|$. 
In general, it is not easy to determine when the graph $\Gamma_{N^{*}}(M)$ is Eulerian or Hamiltonian. Here we consider $M=\mathbb{Z}_{n}$, for some positive integer $n$, and investigate being Eulerian or Hamiltonian (or both) for $\Gamma_{N^{\star}}(M)$.

Lemma 2.20. The followings hold.

(1) [4, Theorem 3.4] If $G$ is a simple graph with $\nu \geq 3$ and $\delta \geq \nu / 2$, where $\nu=|V(G)|$, then $G$ is Hamiltonian.

(2) [4, Theorem 1.4] A connected graph $G$ is Eulerian if and only if it contains no vertices of odd degree.

Example 2.21. Let $M=\mathbb{Z}_{n}$ and $N=2 \mathbb{Z}_{n}$ with $n \geq 8$. Considering Theorem 2.19, $\Gamma_{N^{\star}}(M)$ is $\left|N^{\star}\right|-1$-regular; so $\delta=\left|N^{\star}\right|-1=|N|-2 \geq N / 2$, where $|N|(=n / 2) \geq 4$. Hence by Lemma 2.20, $\Gamma_{N^{\star}}(M)$ is Hamiltonian.

Remark 2.22. If $\Gamma_{N^{\star}}(M)$ is connected, $2 x \notin N^{\star}$ for every $x \in V\left(\Gamma_{N^{\star}}(M)\right)$, and if $\left|N^{\star}\right|$ is an even integer, then $\Gamma_{N^{\star}}(M)$ is Eulerian.

Remark 2.23. Let $M=\mathbb{Z}_{n}$ and $N=k \mathbb{Z}_{n}$ (so, $N=d \mathbb{Z}_{n}$ for $d=(k, n)$ ), and let $\Gamma_{N^{*}}(M)$ be connected.

(1) If $n$ is an odd integer, then $\left|N^{\star}\right|$ is even. Let $2 x \in N^{\star}$ for some $x \in$ $V\left(\Gamma_{N^{\star}}(M)\right)$. Then $2 x=t d$ for some $t \in \mathbb{Z}$. Hence $x \in N$ is not a vertex. So $2 x \notin N^{\star}$ for every $x \in V\left(\Gamma_{N^{\star}}(M)\right)$. Hence, by Lemma 2.20 and Theorem 2.19, $\Gamma_{N^{\star}}(M)$ is Eulerian.

(2) Assume that $n$ and $k$ are even integers; then $d$ is an even integer. By Theorem 2.19, the degree of every vertex $x$ is $\left|N^{\star}\right|$ or $\left|N^{\star}\right|-1$.

(i) Let $n=2^{l}$ for some $l \in \mathbb{N}$. If $d>2$, then there exists at least one vertex $x$ such that $2 x \notin N^{\star}$. So, the degree of the vertex $x$ is $\left|N^{\star}\right|$. Note that $\left|N^{\star}\right|$ is an odd integer. Hence by Lemma 2.20, $\Gamma_{N^{\star}}(M)$ is not Eulerian. If $d=2$, then by Theorem 2.19, $\Gamma_{N^{\star}}(M)$ is a $\left|N^{\star}\right|-1$-regular graph, so it is Eulerian.

(ii) Let $n=2^{l} m$ for some $l, m \in \mathbb{N}$ such that $(2, m)=1$. Since $d=2 m^{\prime} \in N^{\star}$ for some $m^{\prime} \in V\left(\Gamma_{N^{\star}}(M)\right)$, the degree of vertex $m^{\prime}$ is $\left|N^{\star}\right|-1$. Note that $n=t d$ for some $t \in \mathbb{Z}$. If $t$ is an odd integer, then $\left|N^{\star}\right|$ is even. So the degree of $m^{\prime}$ is odd and $\Gamma_{N^{*}}(M)$ is not Eulerian. If $t$ is an even integer, then $\left|N^{\star}\right|$ is odd. We have two cases.

$\left(i^{\prime}\right)$ If $d>2$, there exists at least one vertex $l$ such that $2 l \notin N^{\star}$. Therefore, by Theorem 2.19, $\operatorname{deg}(l)=\left|N^{\star}\right|$, hence $\Gamma_{N^{*}}(M)$ is not Eulerian.

$\left(i i^{\prime}\right)$ If $d=2$, by Theorem 2.19, $\Gamma_{N^{\star}}(M)$ is a $\left|N^{\star}\right|-1$-regular graph and so it is Eulerian. 
(3) Let $n$ be an even integer and $k$ be an odd integer. Since $\left|N^{\star}\right|$ is an odd integer and by part (1), $2 x \notin N^{\star}$ for every $x \in V\left(\Gamma_{N^{\star}}(M)\right), \Gamma_{N^{\star}}(M)$ is not Eulerian.

\section{Independence number and chromatic number of $\Gamma_{N^{*}}(M)$}

One of the interesting computing problems in graph theory is determining the independence number of a graph. Here we obtain the independence number of $\Gamma_{N^{\star}}(M)$ with some special conditions. It is well-known that $\alpha\left(K^{n}\right)=1$.

Lemma 3.1. [4, Theorem 1.7]

(1) A set is independent if and only if its complement is a vertex cover.

(2) The sum of the size of the largest independent set $\alpha(G)$ and the size of a minimum vertex cover $\beta(G)$ is equal to the number of vertices in the graph.

Theorem 3.2. Let $\Gamma_{N^{\star}}(M)$ be connected and let $\nu=|V(G)|$.

(1) If $\operatorname{diam}\left(\Gamma_{N^{\star}}(M)\right)=3$ and $d(m,-m)=3$ for every $m \in V\left(\Gamma_{N^{\star}}(M)\right)$, then $\alpha\left(\Gamma_{N^{*}}(M)\right)=\beta\left(\Gamma_{N^{*}}(M)\right)=\nu / 2$.

(2) If $\operatorname{diam}\left(\Gamma_{N^{*}}(M)\right)=2$ and $2 m \neq 0$ for every $m \in V\left(\Gamma_{N^{*}}(M)\right)$, then $\alpha\left(\Gamma_{N^{*}}(M)\right)=2$ and $\beta\left(\Gamma_{N^{*}}(M)\right)=\nu-2$.

Proof. (1) Choose $x \in V\left(\Gamma_{N^{\star}}(M)\right)$. Put $A_{x}=\left\{-y \in V\left(\Gamma_{N^{\star}}(M)\right) \mid y\right.$ is adjacent to $x\}, A_{x}^{\prime}=\left\{y \in V\left(\Gamma_{N^{\star}}(M)\right) \mid y \neq-x\right.$ and $y$ is not adjacent to $\left.x\right\}$ and $P_{x}=A_{x} \cup A_{x}^{\prime}$. For every $n \in V\left(\Gamma_{N^{\star}}(M)\right)-\{x,-x\}, n \in P_{x}$ or $-n \in P_{x}$.

Claim: $P_{x}$ is an independent set in $\Gamma_{N^{*}}(M)$.

By way of contradiction, suppose there exist $n_{1}, n_{2} \in P_{x}$ that they are adjacent. Since $n_{1}, n_{2} \in P_{x}$, so $n_{1}, n_{2}$ are not adjacent to $x$. We claim that for every vertex $t$ other than $x$ and $-x, t$ is adjacent to either $-x$ or $x$ (but not to both of them, otherwise, $x-t-(-x)$, this yields $d(x,-x)=2)$. Hence, $n_{1}, n_{2}$ are not adjacent to $x$, which implies that $n_{1}, n_{2}$ are adjacent to $-x$.

Suppose there exists $t(\neq x,-x) \in V\left(\Gamma_{N^{\star}}(M)\right)$ such that $t$ is not adjacent to $x$ and $-x$. Since $d(x,-x)=3$, there exists a path $x-m_{1}-m_{2}-(-x)$ in $\Gamma_{N^{*}}(M)$. It is clear that $d(t, x)=d(t,-x)=2$; otherwise, $t$ is adjacent to $x$ or $-x$. So, there exists $l \in V\left(\Gamma_{N^{*}}(M)\right)$ such that $t-l-x$. There is a path $t-l-x-m_{1}-m_{2}-(-x)$ such that $t$ is adjacent to $m_{1}$ (since $m_{1}$ and $x$ are adjacent and $d(t,-x)=2$, so $\left.t \neq-m_{1}\right)$. Hence $t-m_{1}-m_{2}-(-x)$ implies that $t$ and $-x$ are adjacent (since $t \neq x$ ) which is a contradiction. (Therefore, for every vertex $t$, all other vertices except $-t$ are adjacent to $t$ or $-t$.) Since $n_{1}, n_{2}$ are not adjacent to $x$, so $n_{1}, n_{2}$ are adjacent to $-x$ and $n_{1}-(-x)-n_{2}-\left(-n_{1}\right)$ which by assumption $n_{1}$ and $n_{2}$ are adjacent. So, $d\left(n_{1},-n_{1}\right)=2$, a contradiction. This shows that $P_{x}$ is independent. On the other hand, for every $x \neq y \in V\left(\Gamma_{N^{\star}}(M)\right)$, one has 
$\left|P_{x}\right|=\left|P_{y}\right|$. We have to show that $P_{x}$ is the largest independent set in $\Gamma_{N^{*}}(M)$. Suppose there exists an independent set $U$ in $\Gamma_{N^{\star}}(M)$ such that $|U|>\left|P_{x}\right|=|\nu| / 2$, where $\nu=|V(G)|$. So, there exists $g \in V\left(\Gamma_{N^{*}}(M)\right)$ such that $g,-g \in U$. This implies that $U$ is not independent. Hence $P_{x}$ is the largest independent set in $\Gamma_{N^{\star}}(M)$ and $\alpha\left(\Gamma_{N^{\star}}(M)\right)=\beta\left(\Gamma_{N^{\star}}(M)\right)=\nu / 2$ by Lemma 3.1.

(2) Let $\Gamma_{N^{\star}}(M)$ be connected with $\operatorname{diam}\left(\Gamma_{N^{\star}}(M)\right)=2$ and let $2 m \neq 0$ for every $m \in V\left(\Gamma_{N^{\star}}(M)\right)$. Put $G_{x}=\{x,-x\}$ for some $x \in V\left(\Gamma_{N^{\star}}(M)\right)$. We show that $G_{x}$ is the largest independent set in $\Gamma_{N^{\star}}(M)$.

Claim: Every vertex $x$ is adjacent to every other vertex except $-x$.

By way of contradiction, assume that there is $m \in V\left(\Gamma_{N^{*}}(M)\right)$ such that $d(m, x)=$ 2 for some $x(\neq-m) \in V\left(\Gamma_{N^{\star}}(M)\right)$. So there is a path $m-t-x$ in $\Gamma_{N^{\star}}(M)$ for some $t \in V\left(\Gamma_{N^{*}}(M)\right)$; therefore, $(-x)-m-t-x$ such that $x$ and $-m$ are adjacent. Moreover, $d(x,-x)=2$. Hence, there is a path $x-l-(-x)$ in $\Gamma_{N^{*}}(M)$ for some $l \in V\left(\Gamma_{N^{\star}}(M)\right)$. Now, the path $(-m)-x-l-(-x)$ implies that $-m$ is adjacent to $-x$ by Theorem 2.4. So $m$ is adjacent to $x$, a contradiction.

Therefore, $G_{x}$ is the largest independent set in $\Gamma_{N^{\star}}(M)$. In this case $\alpha\left(\Gamma_{N^{\star}}(M)\right)=$ 2 and $\beta\left(\Gamma_{N^{\star}}(M)\right)=\nu-2$ where $\nu=|V(G)|$ by Lemma 3.1.

One of the important aims in graph theory is determining the chromatic number of a given graph. Here we investigate the chromatic number of $\Gamma_{N^{*}}(M)$ in some special cases. It is well-known that $\chi\left(K^{n}\right)=n$ and $\chi(G) \geq \omega(G)$.

Remark 3.3. For all distinct $x, y \in V\left(\Gamma_{N^{*}}(M)\right)$, if $N(x) \cap N(y)=\emptyset$, then it is obvious that $\Gamma_{N^{*}}(M)$ is a perfect matching and $\chi\left(\Gamma_{N^{*}}(M)\right)=2$.

Theorem 3.4. Let $\Gamma_{N^{\star}}(M)$ be connected and non-complete. If there exist two adjacent vertices $x$ and $y$ of $\Gamma_{N^{*}}(M)$ with $N(x) \cap N(y) \neq \emptyset$ or for every two nonadjacent vertices $x$ and $y$ of $\Gamma_{N^{\star}}(M), N(x) \cap N(y) \neq \emptyset$, then $\chi\left(\Gamma_{N^{\star}}(M)\right) \geq 3$.

Proof. Since $\Gamma_{N^{\star}}(M)$ is not complete, there exist non-adjacent vertices $x$ and $y$ in $\Gamma_{N^{*}}(M)$. By assumption, $N(x) \cap N(y) \neq \emptyset$; so $(-y)-x-t-y$ and $l-(-y)-x-t-y-l$ for some $l \in N(y) \cap N(-y)$. Thus $\chi\left(\Gamma_{N^{*}}(M)\right) \geq 3$.

(It should be noted that if $y=-y$, then $\chi\left(\Gamma_{N^{\star}}(M)\right) \geq 3$.)

Theorem 3.5. (1) Let $\Gamma_{N^{*}}(M)$ be connected with $\operatorname{diam}\left(\Gamma_{N^{*}}(M)\right)=3$ and $d(m,-m)=3$ for every $m \in V\left(\Gamma_{N^{\star}}(M)\right)$. Then $\chi\left(\Gamma_{N^{\star}}(M)\right)=2$.

(2) Let $\Gamma_{N^{\star}}(M)$ be connected with $\operatorname{diam}\left(\Gamma_{N^{\star}}(M)\right)=2$ and let $2 m \neq 0$ for every $m \in V\left(\Gamma_{N^{\star}}(M)\right)$. Then $\chi\left(\Gamma_{N^{\star}}(M)\right)=\nu / 2$, where $\nu=|V(G)|$.

Proof. (1) Let $x \in V\left(\Gamma_{N^{\star}}(M)\right)$. Considering our hypothesis and by the proof of part 1 of Theorem 3.2, for every vertex $t$ other than $x$ and $-x, t$ is adjacent to 
either $-x$ or $x$ (but not to both of them, otherwise, $x-t-(-x)$, this implies that $d(x,-x)=2)$. If $t$ is adjacent to $x$, then $t$ is not adjacent to $-x$; so $t \in P_{-x}$, otherwise, $t \in P_{x}$. Hence $P_{x} \cup P_{-x}=V\left(\Gamma_{N^{\star}}(M)\right)$. Now we assign color $a$ to elements of $P_{x}$ and color $b$ to elements of $P_{-x}$. Therefore, $\chi\left(\Gamma_{N^{\star}}(M)\right)=2$.

(2) Let $l_{1} \in V\left(\Gamma_{N^{\star}}(M)\right)$. Here, by the proof of part 2 of Theorem 3.2, every vertex $m$ is adjacent to all other vertices except $-m$. At first we assign color 1 to $l_{1}$ and $-l_{1}$. Choose $l_{i}\left(\neq l_{1},-l_{1}\right) \in V\left(\Gamma_{N^{\star}}(M)\right)$ where $i>1$. Since $l_{i}$ is adjacent to $l_{1}$ and $-l_{1}$ and all of other vertices except $-l_{i}$, we assign color $i$ to $l_{i}$ and $-l_{i}$. Continuing in this manner for remaining vertices of $\Gamma_{N^{\star}}(M)$, one has $\chi\left(\Gamma_{N^{\star}}(M)\right)=\nu / 2$.

Acknowledgment. The authors would like to thank the referee for the valuable suggestions and comments.

\section{References}

[1] A. Abbasi and S. Habibi, The total graph of a commutative ring with respect to proper ideals, J. Korean Math. Soc., 49(1) (2012), 85-98.

[2] A. Abbasi and S. Habibi, The total graph of a module over a commutative ring with respect to proper submodules, J. Algebra Appl., 11(3) (2012), 1250048 (13 pp).

[3] D. F. Anderson and A. Badawi, The total graph of a commutative ring, J. Algebra, 320(7) (2008), 2706-2719.

[4] J. A. Bondy and U. S. R. Murty, Graph Theory with Applications, American Elsevier Publishing Co., Inc., New York, 1976.

[5] S. P. Redmond, An ideal-based zero-divisor graph of a commutative ring, Comm. Algebra, 31(9) (2003), 4425-4443.

\footnotetext{
Ahmad Abbasi (Corresponding Author)

Department of Pure Mathematics

Faculty of Mathematical Sciences

University of Guilan

Rasht, Iran

e-mail: aabbasi@guilan.ac.ir

Leila Hamidian Jahromi

Department of Pure Mathematics

Faculty of Mathematical Sciences

University of Guilan, University Campus 2

Rasht, Iran

e-mail: acz459@yahoo.com
} 